\title{
Multigene families encode the major enzymes of antioxidant metabolism in Eucalyptus grandis L
}

\author{
Felipe Karam Teixeira, Larissa Menezes-Benavente, Vinícius Costa Galvão and Márcia Margis-Pinheiro \\ Universidade Federal do Rio de Janeiro, Departamento de Genética, Laboratório de \\ Genética Molecular Vegetal, Rio de Janeiro, RJ, Brazil.
}

\begin{abstract}
Antioxidant metabolism protects cells from oxidative damage caused by reactive oxygen species (ROS). In plants, several enzymes act jointly to maintain redox homeostasis. Moreover, isoform diversity contributes to the fine tuning necessary for plant responses to both exogenous and endogenous signals influencing antioxidant metabolism. This study aimed to provide a comprehensive view of the major classes of antioxidant enzymes in the woody species Eucalyptus grandis. A careful survey of the FORESTs data bank revealed 36 clusters as encoding antioxidant enzymes: six clusters encoding ascorbate peroxidase (APx) isozymes, three catalase (CAT) proteins, three dehydroascorbate reductase (DHAR), two glutathione reductase (GR) isozymes, four monodehydroascorbate reductase (MDHAR), six phospholipid hydroperoxide glutathione peroxidases (PhGPx), and 12 encoding superoxide dismutases (SOD) isozymes. Phylogenetic analysis demonstrated that all clusters (identified herein) grouped with previously characterized antioxidant enzymes, corroborating the analysis performed. With respect to enzymes involved in the ascorbate-glutathione cycle, both cytosolic and chloroplastic isoforms were putatively identified. These sequences were widely distributed among the different ESTs libraries indicating a broad gene expression pattern. Overall, the data indicate the importance of antioxidant metabolism in eucalyptus.
\end{abstract}

Key words: Eucalyptus, antioxidant metabolism, catalase, ascorbate-gluthatione cycle.

Received: May 31, 2004; Accepted: November 29, 2004.

\section{Introduction}

The exploitation of oxygen reactivity by aerobic metabolism provided a major advantage to aerobic organisms in obtaining chemical energy from biological compounds in a highly efficient manner. However, due to its chemical nature, molecular oxygen often pursues univalent reduction pathways. As a consequence, partially reduced oxygen intermediates, also known as reactive oxygen species (ROS), are produced in many metabolic reactions as byproducts (Scandalios, 2002; Fridovich, 1998). ROS can oxidize all major classes of biological molecules by triggering peroxidative chain reactions, and by leading to oxidative damage to a variety of cellular components. This oxidative stress may ultimately cause cell death. However, ROS also fulfill many important cellular functions. In plants, some of the processes involved are regulation of gene expression, cell cycle, programmed cell death, hypersensitive response,

Send correspondence to Márcia Margis-Pinheiro. Universidade Federal do Rio de Janeiro, Departamento de Genética, Laboratório de Genética Molecular Vegetal, 21944-970, Rio de Janeiro, RJ, Brazil. E-mail: margism@biologia.ufrj.br. and senescence (Bowler and Fluhr, 2000; Dat et al., 2000; Jones, 2001; Scandalios, 2002).

Antioxidant metabolism evolved to maintain cell redox homeostasis and to protect cells against the potentially hazardous effects of ROS (Scandalios, 2002; Fridovich, 1998). Besides scavenging ROS produced during the regular metabolic processes, antioxidant metabolism also has a major role in plant protection against stressful environmental conditions, which cause an increase in ROS production and accumulation (Dat et al., 2000). Both enzymatic and non-enzymatic components participate in various reactions involved in ROS detoxification and damage repair. Many antioxidant enzymes act jointly to keep a healthy cell redox status at the different cellular compartments (Figure 1). Among these, superoxide dismutases (SOD, EC 1.15.1.1) constitute a frontline defense, by removing the radical superoxide, $\mathrm{O}_{2}^{-}$. The resulting hydrogen peroxide, $\mathrm{H}_{2} \mathrm{O}_{2}$, is further detoxified by catalases (CAT, EC 1.11.1.6). $\mathrm{H}_{2} \mathrm{O}_{2}$ is also removed by the action of peroxidases, which require a reducing substrate as an electron donor (Noctor and Foyer, 1998). In plants, among the reducing substrate dependent peroxidases, ascorbate peroxidases (APx, EC 1.11.1.11) 


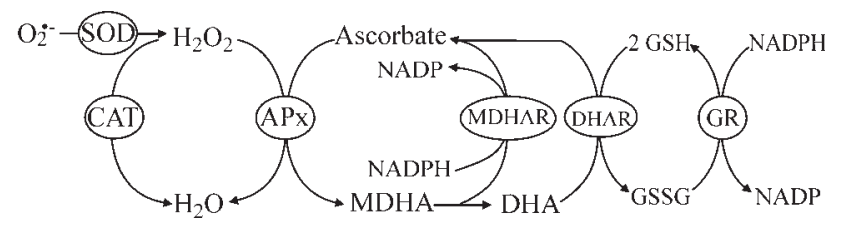

Figure 1 - Reactive oxygen species (ROS) scavenging pathways in plants. Not all reactions are depicted stoichiometrically. Abbreviation: APx Ascorbate peroxidase; CAT - Catalase; DHA - Dehydroascorbate; DHAR - Dehydroascorbate reductase; MDHA - Monodehydroascorbate; MDHAR - Monodehydroascorbate reductase; GR - Glutathione reductase; GSH - Reduced glutathione; GSSG - Glutathione disulphide; SOD - Superoxide dismutase.

are the major enzymes involved in intracellular $\mathrm{H}_{2} \mathrm{O}_{2}$ removal. These enzymes utilize ascorbate (Asc) as an electron donor. Compared to CAT, APx possess a higher affinity towards $\mathrm{H}_{2} \mathrm{O}_{2}$, but have a lower processing rate (Noctor and Foyer, 1998). Among damage repair systems, phospholipid hydroperoxide glutathione peroxidases (PhGPx, EC 1.11.1.12) have a prominent role in removal of lipid peroxides produced by oxidation of biological membranes (Eshdat et al., 1997).

APx catalyzes the first step of the ascorbate-glutathione cycle, a major antioxidant route operating in plant cells. In this cycle, ascorbate and glutathione are employed as reducing power to detoxify $\mathrm{H}_{2} \mathrm{O}_{2}$. Oxidized ascorbate and glutathione are not expended, but further rescued at the expense of ATP and NAD(P)H (Figure 1). Besides APx, monodehydroascorbate reductase (MDHAR, EC 1.6.5.4), dehydroascorbate reductase (DHAR, EC 1.8.5.1), and glutathione reductase (GR, EC 1.8.1.7) also catalyze important steps of this cycle (Alscher et al.., 1997; Noctor and Foyer, 1998). The main role of the ascorbate-glutathione cycle is chloroplast protection against oxidative damage, although it also operates in the cytoplasm. It has also been suggested that this cycle operates in other organelles such as peroxisomes and mitochondria (Jiménez et al., 1997). However, only circumstantial evidence has been presented so far and further evidence is necessary to confirm this hypothesis.

In plants, multigene families encode the major antioxidant enzymes. This confers a great adaptive advantage by allowing a differential regulation of each gene family member in response to different endogenous (tissue and developmental) and exogenous (e.g., environmental) stimuli. SOD, CAT, and APx families have been studied in some detail in maize, arabidopsis, tobacco, and sugarcane (Willekens et al., 1994; Jespersen et al., 1997; McClung, 1997; Scandalios, 1997; Scandalios et al., 1997; Van Camp et al., 1997; Netto, 2001; Shigeoka et al., 2002). The present study aimed to provide a comprehensive analysis of the major antioxidant enzymes encoding genes in a single plant species, using a genome-based analysis of the woody species Eucalyptus grandis. This study is part of the Brazilian FORESTs project, whose goal is to decipher the expressed genome of this plant. Besides understanding the underlying mechanisms involved in cell redox homeostasis, the study of antioxidant metabolism can aid in proposing new strategies for plant and crop improvement to combat stressful conditions.

\section{Material and Methods}

\section{Retrieval of Eucalyptus sequences}

Clusters encoding antioxidant enzymes in Eucalyptus were identified by extensive searches using the TBLASTN program (Altschul et al., 1990) against the FORESTs cluster data bank (https://forests.esalq.usp.br/). Initially, protein sequences encoding antioxidant enzymes from different organisms were recovered from the National Center for Biotechnology Information (NCBI) data bank (http://www.ncbi.nlm.nih.gov/) by keyword searches. Then these sequences were used as probes in the TBLASTN analyses. The resulting clusters with an E-value lower than $\mathrm{e}^{-10}$ were analyzed individually by manual inspection of the six-frame translation products generated in the BCM search launcher (http://searchlauncher.bcm.tmc. edu/). The ProDom (http://prodes.toulouse.inra.fr/prodom/ current/html/home.php) and PROSITE (http://www. expasy.org/prosite/) databases were used in the identification of the clusters.

\section{FORESTs clusters libraries}

All Eucalyptus sequences used in this work were obtained from the FORESTs project and are derived from ESTs libraries specific to different tissues, organs, or growth conditions (for detailed information see https://forests.esalq.usp.br/).

\section{Sequence alignments}

Multiple sequence alignments were constructed using the program ClustalW 1.8 (Higgins and Sharp, 1988) at the European Bioinformatics Institute server (http://www.ebi. ac.uk/clustalw/). Protein sequence alignments were performed with the following parameters: "Gap Opening penalty" = 10.0, "Gap Extension penalty" $=0.05$ and BLOSUM (Henikoff) protein weight matrices. The multiple alignments were inspected by eye and edited using GeneDoc program Version 2.6.002 (Nicholas et al., 1997).

\section{Phylogenetic constructions}

The molecular evolutionary and phylogenetic analyses were conducted using the software Molecular Evolutionary Genetics Analysis (MEGA) Version 2.1 (Kumar et al., 2001). The molecular distances of the aligned sequences were calculated according to the parameter of p-distance, and the phylogenetic trees were constructed using the Neighbor-Joining method with pairwise deletion. The phylogeny test used was the Interior Branch Test with 1000 Replications. 


\section{Protein sequence analyses}

The identification of putative transmembrane domains was accomplished by using the SOSUI program Version 1.0 (Hirokawa et al., 1998) present in the BCM search launcher. Protein sorting and subcellular localization predictions were performed according to ProtComp program Version 5 (http://www.softberry.com/) and PSORT software Version 6.4 (http://psort.nibb.ac.jp/) (Nakai and Horton, 1999).

\section{Results and Discussion}

\section{Identification of FORESTs antioxidant enzyme clusters}

In order to control the cellular level of ROS, plants have evolved the antioxidant system that scavenge oxygen free radicals and protect cells against oxidative damage (Scandalios, 2002). Antioxidant enzymes and their corresponding genes have been studied in many plants, although no systematic study has been made to identify these enzymes in a single plant species. This work aimed to investigate this issue by taking advantage of the FORESTs database, which contains about 33,000 ESTs clusters distributed in several libraries. In order to identify Eucalyptus antioxidant enzymes encoding genes, TBLASTN searches in FORESTs clusters database were conducted using aminoacid sequences of antioxidant enzymes from different organisms, recovered from the NCBI data bank, as a probe. The overall analysis, with a cut-off value of $\mathrm{e}^{-10}$, allowed the identification of 116 different clusters, which were analyzed individually. 36 Eucalyptus EST-clusters highly similar to different classes of antioxidant enzyme encoding genes were identified: six clusters encoding APx isozymes, three CAT proteins, three DHAR, two GR isozymes, four MDHAR, six PhGPx, and 12 encoding SOD isozymes. Some of these clusters are incomplete and encode partial proteins. The clusters identified were used as probes in the TBLASTN program against the NCBI protein database and were analyzed by protein sorting prediction and subcellular localization programs. Clusters accession numbers in FORESTs database, BLAST results, and putative localization of protein products based on subcellular localization prediction programs are summarized in Table 1 .

\section{Eucalyptus superoxide dismutase gene family}

Superoxide dismutases are ubiquitous enzymes that catalyze the conversion of $\mathrm{O}_{2}^{-}$to $\mathrm{H}_{2} \mathrm{O}_{2}$ and are found in almost all oxygen-consuming organisms. They are classified according to their metal cofactor: copper-zinc $(\mathrm{Cu}, \mathrm{Zn})$, manganese $(\mathrm{Mn})$ or iron $(\mathrm{Fe})$. Mn-SODs are generally found in mitochondria and Fe-SODs in chloroplasts. On the other hand, cytosolic and chloroplastic $\mathrm{Cu}, \mathrm{Zn}$-SODs isoforms are broadly described (Scandalios, 1997). Extracellular $\mathrm{Cu}, \mathrm{Zn}-\mathrm{SODs}$ are common in animals. In plants, extracellular $\mathrm{Cu}, \mathrm{Zn}-\mathrm{SOD}$ with high isoelectric points were described in pine (Karpinska et al., 2001) and in the hybrid aspen (Schinkel et al., 2001).

Twelve Eucalyptus SOD encoding clusters were identified. The phylogenetic and subcellular prediction analyses revealed that two clusters correspond to putative mitochondrial Mn-SOD and five encoding putative chloroplastic Fe-SOD isoforms. Among the five clusters encoding putative $\mathrm{Cu}, \mathrm{Zn}$-SOD, two are putative cytosolic isoforms, two encode putative chloroplastic proteins, and one cluster corresponds to a putative extracellular isoform (Table 1 and Figure 2).

Surprisingly, we have identified a larger number of Eucalyptus Fe-SOD genes in comparison to other plants. Only one Fe-SOD isoform was described in tobacco (Van Camp et al., 1997) and three were found in Arabidopsis (Kliebenstein et al., 1998). Moreover, a cluster corresponding to a putative extracellular $\mathrm{Cu}, \mathrm{Zn}$-SOD similar to the high isoelectric point isoforms from pine and hybrid aspen (Karpinska et al., 2001; Schinkel et al., 2001) was found in the Eucalyptus FORESTs data bank (Figure 2). Thus, all SOD isoforms described in plants were identified in Eucalyptus. To our knowledge, this is the largest SOD gene family described so far.

A preliminary investigation was conducted into the expression pattern of the SOD genes in Eucalyptus. Eucalyptus SOD isoforms are distributed in almost all FORESTs libraries (Table 2). Three Fe-SOD chloroplastic encoding clusters (EGCBSL4285B02.g, EGMCFB1066 G03.g, and EGJFLV2207E09.g) and one corresponding to a $\mathrm{Cu}, \mathrm{Zn}-\mathrm{SOD}$ cytosolic isoform (EGCCST6227F01.g) are present in a single library. Moreover, two Fe-SOD isoforms (EGEZRT3005F09.g and EGEQRT3100G07.b) were the most frequent clusters found within the libraries. Taken together, the number of Eucalyptus clusters encoding SOD and their large distribution in FORESTs ESTs libraries reveal the crucial role of these enzymes in ROS scavenging in different tissues and growth conditions.

\section{Catalase genes}

Catalase plays a central role in the antioxidant defense mechanisms and is found in a wide range of organisms from aerobic bacteria to higher plants and animals. Plant catalases are encoded by a small gene family, usually three isozyme encoding genes in a single species, as previously described in maize (Scandalios, 1965), tobacco (Havir and McHale, 1987; Willekens et al., 1994), cottonseed (Ni et al., 1990), Arabidopsis (Frugoli et al., 1996), and rice (Iwamoto et al., 1998). These enzymes catalyze the conversion of $\mathrm{H}_{2} \mathrm{O}_{2}$ to $\mathrm{H}_{2} \mathrm{O}$ and $\mathrm{O}_{2}$ and are preferentially present in peroxisomes (Noctor and Foyer, 1998).

Similarly to those observed in other plants, we have identified three Eucalyptus clusters encoding putative catalases (Table 1). The subcellular localization prediction indicates that these clusters encode putative peroxisomal 
Table 1 - Antioxidant enzyme encoding clusters in the FORESTs databank ( ${ }^{1}$ Incomplete clusters).

\begin{tabular}{|c|c|c|c|}
\hline FOREST cluster & Best Hit & e-value & $\begin{array}{l}\text { Putative subcellular } \\
\text { localization }\end{array}$ \\
\hline \multicolumn{4}{|l|}{ APx } \\
\hline EGEZLV2207C08.g & T09125 - L-ascorbate peroxidase (EC 1.11.1.11) - spinach & e-100 & Cytosolic \\
\hline EGEZRT4203A05.g & AAD41404 - cytosolic ascorbate peroxidase [Fragaria $x$ ananassa] & e-116 & Cytosolic \\
\hline EGRFST6257D01.g & CAA56340 - ascorbate peroxidase [Arabidopsis thaliana] & e-105 & Cytosolic \\
\hline EGEZLV2203G08.g & T09845 - L-ascorbate peroxidase (EC 1.11.1.11), glyoxysomal - upland cotton & e-122 & Peroxisome \\
\hline EGEQFB1200E08.g & BAA22196 - stromal ascorbate peroxidase [Cucurbita $\mathrm{cv}$. Kurokawa Amakuri] & e-154 & Chloroplast \\
\hline EGRFRT3023B08.g ${ }^{1}$ & BAA78552 - thylakoid-bound ascorbate peroxidase [Nicotiana tabacum] & $7 e-89$ & Chloroplast \\
\hline \multicolumn{4}{|l|}{ CAT } \\
\hline EGEQLV1201F10.g & CAD42908 - catalase [Prunus persica] & 0.0 & Peroxisome \\
\hline EGEQFB1001H11.g & AAL83720 - catalase [ Vitis vinifera $]$ & 0.0 & Peroxisome/mb \\
\hline EGEZLV1203E04.g & O24339 - Catalase [Soldanella alpina] & 0.0 & Peroxisome \\
\hline \multicolumn{4}{|l|}{ DHAR } \\
\hline EGEPFB1249A11.g & AAL71857 - dehydroascorbate reductase [Nicotiana tabacum] & $5 e-84$ & Cytosolic \\
\hline EGQHST6232H12.g ${ }^{1}$ & NP_177662 - dehydroascorbate reductase, putative [Arabidopsis thaliana] & $1 \mathrm{e}-28$ & Cytosolic \\
\hline EGEZSL 7225G03.g ${ }^{1}$ & AAN04048 - dehydroascorbate reductase [Brassica juncea] & $8 \mathrm{e}-61$ & Chloroplast \\
\hline \multicolumn{4}{|l|}{ GR } \\
\hline EGEQRT3201H09.g & Q43621 - Glutathione reductase, cytosolic (GR) & 0.0 & Cytosolic \\
\hline EGEQRT6002D01.. ${ }^{1}$ & P80461 - Glutathione reductase, chloroplast precursor (GR) & $\mathrm{e}-126$ & Chloroplast \\
\hline \multicolumn{4}{|l|}{ MDHAR } \\
\hline EGEZST2206C02.g & CAC82727 - monodehydroascorbate reductase [Mesembryanthemum crystallinum] & 0.0 & Cytosolic \\
\hline EGEQRT3201G06.g ${ }^{1}$ & JU0182 - monodehydroascorbate reductase (NADH2) (EC 1.6.5.4) - cucumber & $6 e-98$ & Cytosolic \\
\hline EGEZLV1202F11.g & NP_189420 - monodehydroascorbate reductase, putative [Arabidopsis thaliana] & 0.0 & Membrane bound \\
\hline EGSBRT3312H11. $\mathrm{g}^{1}$ & NP_849839 - monodehydroascorbate reductase, putative [Arabidopsis thaliana] & 0.0 & Chloroplast \\
\hline \multicolumn{4}{|l|}{ PHGPx } \\
\hline EGABSL1068A12.g & NP_566128 - glutathione peroxidase, putative [Arabidopsis thaliana] & $5 e-70$ & Cytosolic \\
\hline EGACRT3318G10.g & S33618 - glutathione peroxidase (EC 1.11.1.9) - sweet orange & $7 \mathrm{e}-76$ & Cytosolic \\
\hline EGBFST7278B12.g & NP_564813 - glutathione peroxidase, putative [Arabidopsis thaliana] & $9 e-69$ & Cytosolic \\
\hline EGCCFB1223B07.g & AAC78466 - glutathione peroxidase [Zantedeschia aethiopica] & $2 \mathrm{e}-82$ & Chloroplast \\
\hline EGEPFB1249H12.g & NP_192897 - glutathione peroxidase, putative [Arabidopsis thaliana] & $3 e-78$ & Chloroplast \\
\hline EGQHSL1102C02.g & AAL40914 - phospholipid hydroperoxide glutathione peroxidase [Momordica charantia] & $2 \mathrm{e}-79$ & Chloroplast \\
\hline \multicolumn{4}{|l|}{ SOD } \\
\hline \multicolumn{4}{|l|}{ Iron } \\
\hline EGCBSL4285B02. ${ }^{1}$ & NP_197722 - superoxide dismutase [Fe] / iron superoxide dismutase 3 (FSD3) [Arabidopsis thaliana] & $4 \mathrm{e}-70$ & Chloroplast \\
\hline EGEZRT3005F09.g & CAE22480 - superoxide dismutase [Fe] [Lycopersicon esculentum] & $5 e-83$ & Chloroplast \\
\hline EGEQRT3100G07.b & A39267 - superoxide dismutase (EC 1.15.1.1) $(\mathrm{Fe})$ - curled-leaved tobacco & $5 \mathrm{e}-73$ & Chloroplast \\
\hline EGMCFB1066G03.g & AAQ18699 - iron superoxide dismutase [Lycopersicon esculentum] & $1 \mathrm{e}-62$ & Chloroplast \\
\hline EGJFLV2207E09.g ${ }^{1}$ & NP_199923 - superoxide dismutase [Fe], putative / iron superoxide dismutase [Arabidopsis thaliana] & $3 e-88$ & Chloroplast \\
\hline \multicolumn{4}{|l|}{ Manganese } \\
\hline EGQHST2018D10.g & P11796 - Superoxide dismutase [Mn], mitochondrial precursor & $2 \mathrm{e}-99$ & Mitochondria \\
\hline EGBFFB1039E04.b & Q9SM64 - Superoxide dismutase [Mn], mitochondrial precursor & e-106 & Mitochondria \\
\hline \multicolumn{4}{|l|}{ Copper/Zinc } \\
\hline EGCCST6227F01.g & AAD01604 - cytoplasmic superoxide dismutase 1 [Populus tremuloides] & $6 e-60$ & Cytosolic \\
\hline EGEQLV2200A04.g & CAE54085 - superoxide dismutase [Fagus sylvatica $]$ & $3 e-56$ & Cytosolic \\
\hline EGBGRT3205E07.g & CAC33847 - putative CuZn-superoxide dismutase [Populus tremula x Populus tremuloides] & $2 \mathrm{e}-61$ & Extracellular - high pl \\
\hline EGUTSL1027E08.g & CAC33844 - putative CuZn-superoxide dismutase [Populus tremula x Populus tremuloides] & $2 \mathrm{e}-64$ & Chloroplast \\
\hline EGEQLV2003C09.g & O65199 - Superoxide dismutase [Cu-Zn], chloroplast precursor & $2 \mathrm{e}-54$ & Chloroplast \\
\hline
\end{tabular}

mb: membrane bound. 
Table 2 - Frequency of antioxidant enzymes encoding clusters in FORESTs libraries.

\begin{tabular}{|c|c|c|c|c|c|c|c|c|c|c|c|c|c|c|c|c|c|c|c|c|}
\hline \multirow[t]{2}{*}{ FORESTs cluster } & \multicolumn{19}{|c|}{ Library } & \multirow[t]{2}{*}{ Total } \\
\hline & BK1 & CL1 & CL2 & FB1 & LV1 & LV2 & LV3 & RT3 & RT6 & SL1 & SL4 & SL5 & SL6 & SL7 & SL8 & ST2 & ST6 & ST7 & WD2 & \\
\hline \multicolumn{21}{|l|}{ APx } \\
\hline EGEZLV2207C08.g & 1 & 2 & 2 & 2 & 1 & 8 & - & 1 & 1 & 5 & 2 & 1 & - & - & 8 & - & 2 & 1 & - & 37 \\
\hline EGEZRT4203A05.g & - & 2 & - & - & - & 3 & 1 & 2 & 3 & 3 & 5 & 2 & - & 1 & - & 5 & 8 & - & 2 & 37 \\
\hline EGRFST6257D01.g & - & - & - & - & - & - & - & 1 & - & - & - & - & 1 & 2 & - & 1 & 1 & - & - & 6 \\
\hline EGEZLV2203G08.g & - & 2 & - & 3 & - & 3 & 3 & - & 1 & 3 & 2 & - & - & - & - & 2 & - & - & - & 19 \\
\hline EGEQFB1200E08.g & - & 1 & - & 4 & - & 1 & - & - & 3 & - & 4 & 1 & - & - & - & 1 & - & - & - & 15 \\
\hline EGRFRT3023B08.g & - & 2 & 1 & 1 & - & - & - & 3 & - & - & 1 & - & - & - & - & 3 & 1 & - & - & 12 \\
\hline \multicolumn{21}{|l|}{ CAT } \\
\hline EGEQLV1201F10.g & - & 2 & 2 & 2 & 6 & 7 & 16 & 1 & - & 9 & 15 & 9 & 1 & 1 & 3 & 1 & - & - & - & 75 \\
\hline EGEQFB1001H11.g & 5 & 90 & 9 & 17 & - & 4 & 9 & 41 & 31 & 16 & 61 & 23 & 8 & 15 & 20 & 34 & 50 & 14 & 11 & 458 \\
\hline EGEZLV1203E04.g & - & 2 & 2 & 4 & 1 & 1 & 1 & 3 & 2 & 3 & - & 5 & - & 3 & 2 & 1 & 3 & 1 & 3 & 37 \\
\hline \multicolumn{21}{|l|}{ DHAR } \\
\hline EGEPFB1249A11.g & - & - & - & 1 & - & 4 & - & - & 3 & - & - & - & - & - & - & - & - & - & 1 & 9 \\
\hline EGQHST6232H12.g & - & - & - & - & - & - & - & - & - & - & - & - & - & - & - & - & - & 1 & - & 1 \\
\hline EGEZSL7225G03.g & - & - & - & - & - & - & - & - & - & - & 1 & - & - & 1 & - & - & - & - & - & 2 \\
\hline \multicolumn{21}{|l|}{ GR } \\
\hline EGEQRT3201H09.g & - & - & - & - & - & - & - & 2 & 4 & 2 & 2 & - & - & 1 & - & 2 & 2 & - & 3 & 18 \\
\hline EGEQRT6002D01.g & - & - & - & - & - & - & - & - & 1 & - & - & - & - & - & - & 1 & - & - & - & 2 \\
\hline \multicolumn{21}{|l|}{ MDHAR } \\
\hline EGEZST2206C02.g & 2 & 6 & - & 1 & - & 2 & 2 & 3 & 4 & 1 & 10 & 4 & 1 & 3 & - & 8 & 12 & - & 3 & 62 \\
\hline EGEQRT3201G06.g & - & - & - & - & - & - & 2 & 1 & 1 & 2 & - & - & 1 & - & - & - & 1 & - & 2 & 10 \\
\hline EGEZLV1202F11.g & - & 1 & - & 1 & 1 & 2 & 1 & 2 & - & 4 & 2 & 1 & - & 1 & - & - & - & - & - & 16 \\
\hline EGSBRT3312H11.g & - & - & - & - & - & - & - & 6 & 1 & - & 2 & 1 & - & - & - & - & 1 & - & - & 11 \\
\hline \multicolumn{21}{|l|}{ PHGPx } \\
\hline EGABSL1068A12.g & - & - & - & - & - & - & - & 1 & 1 & 2 & - & - & - & - & - & - & - & - & - & 4 \\
\hline EGACRT3318G10.g & - & - & 1 & 1 & - & 4 & - & 1 & - & - & - & 1 & - & - & - & - & - & - & - & 8 \\
\hline EGBFST7278B12.g & - & - & - & - & - & - & - & - & - & - & - & - & - & - & - & - & - & 1 & - & 1 \\
\hline EGCCFB1223B07.g & - & 1 & - & 4 & - & - & - & 1 & - & - & - & 1 & 1 & - & - & - & - & - & - & 8 \\
\hline EGEPFB1249H12.g & - & 4 & 3 & 2 & - & 3 & - & - & 1 & 2 & 1 & - & - & - & - & 3 & 1 & - & - & 20 \\
\hline EGQHSL1102C02.g & - & - & - & - & - & - & - & - & - & 2 & - & 3 & - & - & - & - & 2 & - & - & 7 \\
\hline \multicolumn{21}{|l|}{ SOD } \\
\hline \multicolumn{21}{|l|}{ Iron } \\
\hline EGCBSL4285B02.g & - & - & - & - & - & - & - & - & - & - & 1 & - & - & - & - & - & - & - & - & 1 \\
\hline EGEZRT3005F09.g & - & 4 & 1 & - & - & 1 & 4 & 6 & 2 & 2 & - & - & - & 1 & - & - & - & 3 & - & 24 \\
\hline EGEQRT3100G07.b & - & 19 & 1 & - & - & - & 3 & 5 & 2 & 1 & 1 & - & - & - & - & - & 1 & 3 & - & 36 \\
\hline EGMCFB1066G03.g & - & - & - & 1 & - & - & - & - & - & - & - & - & - & - & - & - & - & - & - & 1 \\
\hline EGJFLV2207E09.g & - & - & - & - & - & 1 & - & - & - & - & - & - & - & - & - & - & - & - & - & 1 \\
\hline \multicolumn{21}{|l|}{ Manganese } \\
\hline EGQHST2018D10.g & - & 5 & - & - & - & - & - & - & - & - & - & 2 & - & - & - & 4 & - & - & 1 & 12 \\
\hline EGBFFB1039E04.b & - & - & - & 7 & - & - & - & - & 3 & 1 & - & - & - & - & 1 & 1 & 1 & - & 1 & 15 \\
\hline \multicolumn{21}{|l|}{ Copper/Zinc } \\
\hline EGCCST6227F01.g & - & - & - & - & - & - & - & - & - & - & - & - & - & - & - & - & 1 & - & - & 1 \\
\hline EGEQLV2200A04.g & - & - & - & 3 & - & 4 & - & - & - & - & 2 & - & - & 2 & - & - & - & - & 1 & 12 \\
\hline EGBGRT3205E07.g & - & - & - & 1 & - & - & - & 1 & - & 1 & - & - & - & - & - & - & - & - & - & 3 \\
\hline EGUTSL1027E08.g & - & - & - & - & - & 3 & - & - & - & 3 & 2 & - & - & - & - & - & - & - & - & 8 \\
\hline EGEQLV2003C09.g & - & - & - & - & - & 4 & - & - & - & 1 & - & - & - & - & 1 & 2 & - & - & - & 8 \\
\hline $\begin{array}{l}\text { Total number of reads } \\
\text { of each library }\end{array}$ & 1052 & 9998 & 2535 & 12275 & 678 & 7352 & 4341 & 13252 & 6877 & 6182 & 6718 & 7165 & 1217 & 4120 & 2035 & 11032 & 12558 & 2728 & 10224 & 122339 \\
\hline
\end{tabular}




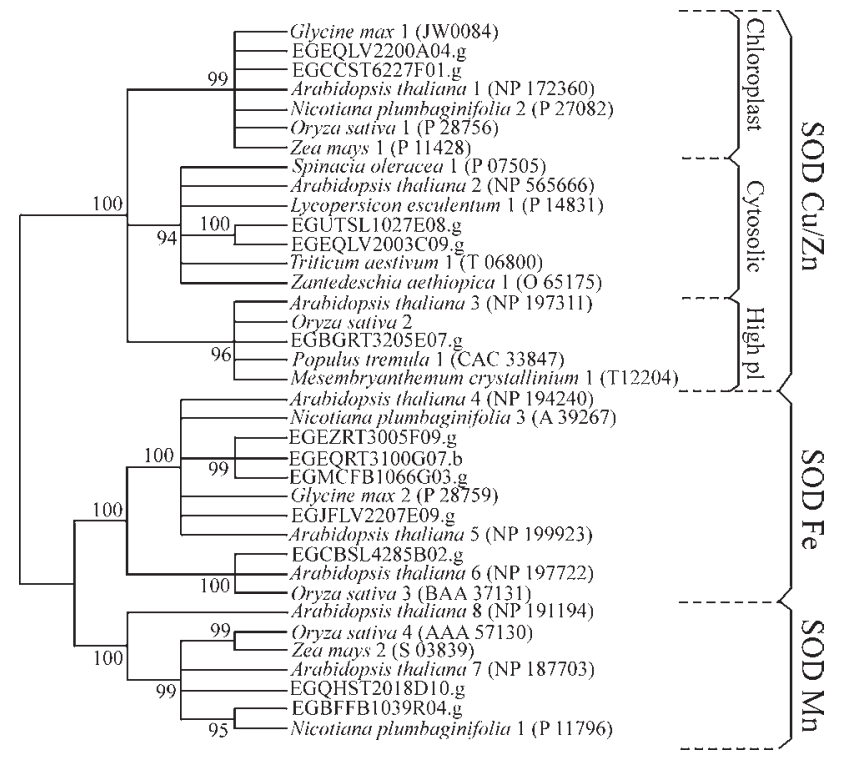

Figure 2 - Phylogenetic tree of SOD proteins. Phylogenetic analyses were conducted using the software MEGA Version 2.1. Molecular distances were calculated using the parameter of p-distance, and the trees were constructed using the Neighbor-Joining method with pairwise deletion. The test of phylogeny used was the Interior Branch Test with 1000 replications. Confidence test values $90 \%$ are shown. Protein sequences are identified by their accession number at NCBI.

proteins. Additionally, the cluster EGEQFB1001H11.g harbors a putative hydrophobic transmembrane domain suggesting that this protein is attached to the peroxisome membrane. The phylogenetic analysis shows Eucalyptus CAT encoding clusters grouping with other catalase proteins (Figure 3), corroborating the initial prediction. The yeast cytochrome-c peroxidase (accession number NP 012992) was included in the phylogenetic analysis as outgroup.

CAT Eucalyptus transcripts are represented in almost all FORESTs libraries (Table 2). When compared to other antioxidant enzymes, CAT encoding clusters present a larger difference of frequency and are the most represented class of antioxidant enzyme encoding clusters in different FORESTs libraries. Besides being the most expressed cluster among the antioxidant enzymes encoding clusters identified herein, the cluster EGEQFB1001H11.g was also the second most expressed cluster among all clusters present in FORESTs database. Altogether, these results demonstrate the major importance of catalases in Eucalyptus antioxidant metabolism.

\section{Eucalyptus ascorbate-glutathione cycle}

Tight control of cellular $\mathrm{H}_{2} \mathrm{O}_{2}$ levels is important to avoid oxidative damage of cellular components and to maintain the regulation of various cell responses. The ascorbate-glutathione cycle comprises several reactions that result in the scavenging of $\mathrm{H}_{2} \mathrm{O}_{2}$, using the reducing power of ascorbate and glutathione (Figure 1). APx, MDHAR, DHAR, and GR catalyze key steps of this cycle

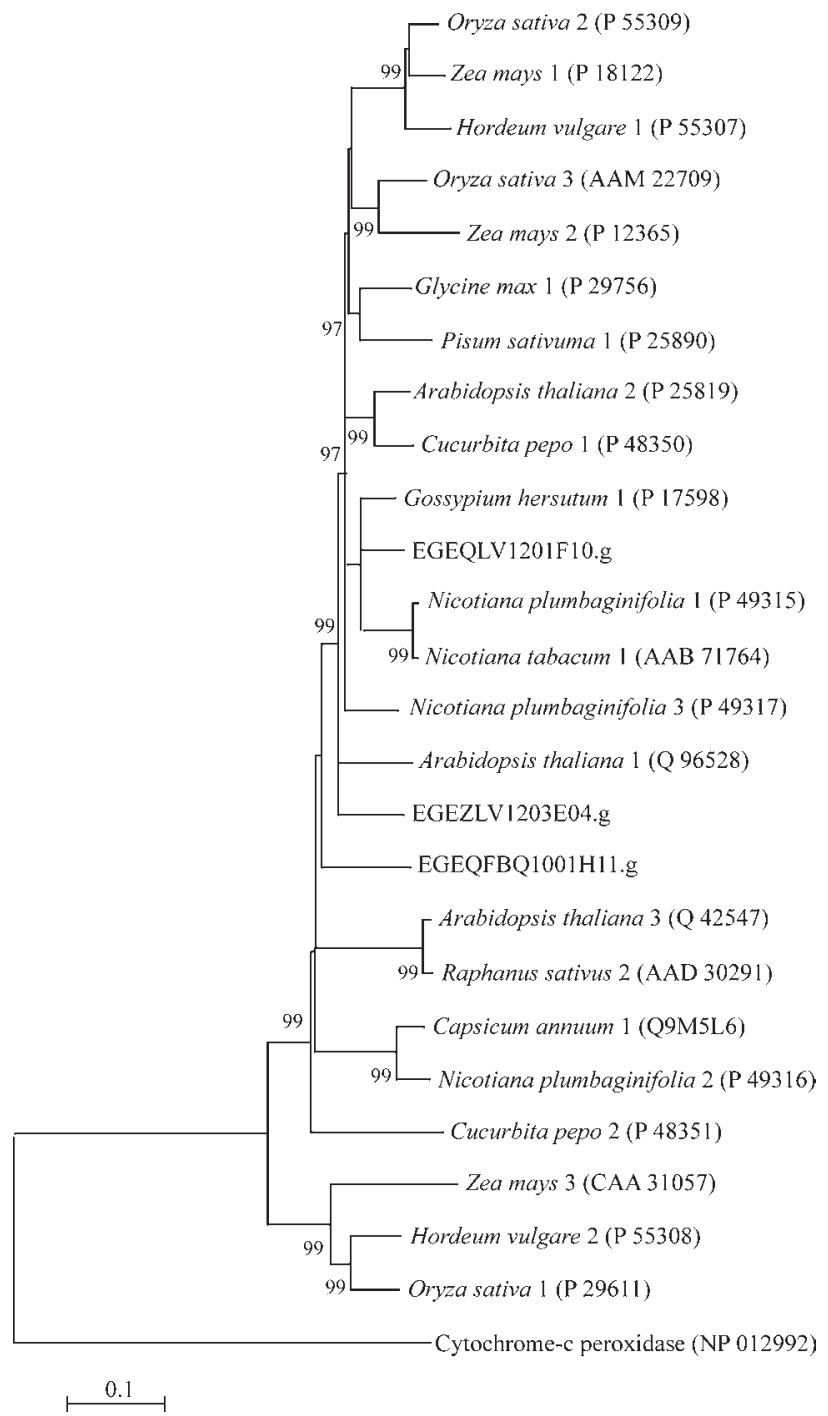

Figure 3 - Phylogenetic tree of CAT proteins. Phylogenetic analyses were conducted as described in Figure 2.

(Noctor and Foyer, 1998). We identified 15 Eucalyptus clusters encoding enzymes of the ascorbate-glutathione cycle (Table 1).

Eucalyptus clusters encoding APx were analyzed by subcellular localization prediction programs. Among the six isoforms, three were putatively identified as cytosolic clusters, one as a putative peroxisomal protein, and two putatively associated with chloroplasts (Table 1). The phylogenetic analysis presented in Figure 4 corroborates the initial prediction of the subcellular localization of the APX in Eucalyptus. The closely related yeast cytochrome-c peroxidase (accession number NP012992) was included in the phylogenetic analysis as an out-group (Patterson and Poulos, 1995).

The putative peroxisomal Eucalyptus APx (EGEZLV 2203G08.g) harbors a hydrophobic transmembrane domain, adjacent to a conserved positively charged 5-residue domain at the $\mathrm{C}$-terminal, previously described in cotton- 


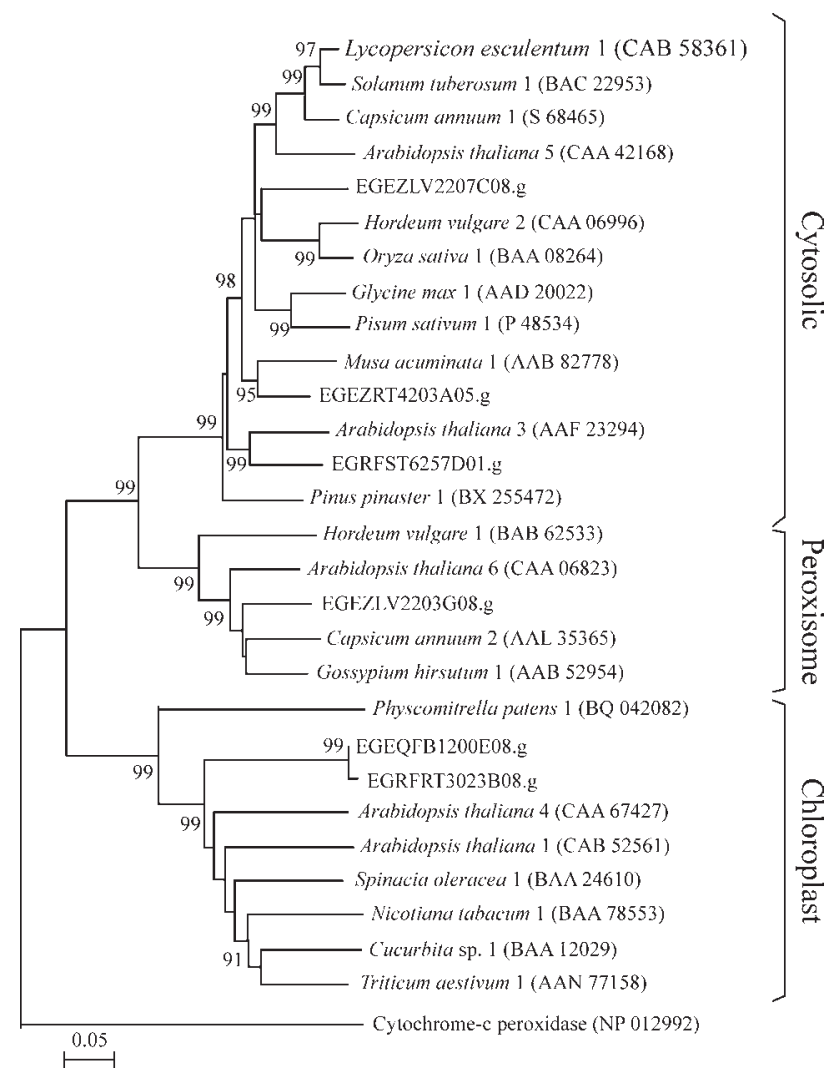

Figure 4 - Phylogenetic tree of APx proteins. Phylogenetic analyses were conducted as described in Figure 2.

seed peroxisomal APx isoform (Mullen and Trelease, 2000). Similarly, the eucalyptus putative chloroplastic isoforms encoded by clusters EGEQFB1200E08.g and EGRFRT3023B08.g are preceded by an N-terminal extension region characteristic of chloroplastic sorting peptide sequences, which is processed in mature chloroplastic proteins (Madhusudhan et al., 2003). Although cluster EGEQFB1200E08.g encodes a putative stromal isoform, no final conclusion can be made about cluster EGRFRT3023B08.g subcellular localization, since this is a 3 '-incomplete cluster.

In the second step of the ascorbate-glutathione cycle, MDHAR catalyzes the reduction of monodehydroascorbate radicals to ascorbate using $\mathrm{NAD}(\mathrm{P}) \mathrm{H}$ as the electron donor (Hossain et al., 1998) (Figure 1). MDHAR cDNAs were cloned from cucumber (Sano and Asada, 1994), garden pea (Murthy and Zilinskas, 1994), Arabidopsis (Obara et al., 2002), and tomato (Grantz et al., 1995). Four Eucalyptus clusters were identified as encoding MDHAR isoforms. Phylogenetic and subcellular prediction analyses showed that two clusters correspond to putative cytosolic isoforms, one to a membrane-bound isoform, and one encodes a putative chloroplastic isoform (Table 1 and Figure 5). An N-terminal chloroplastic sorting peptide sequence present in a MDHAR chloroplastic isoform was previously described in Arabidopsis thaliana (Obara et al., 2002). Unfortunately, cluster

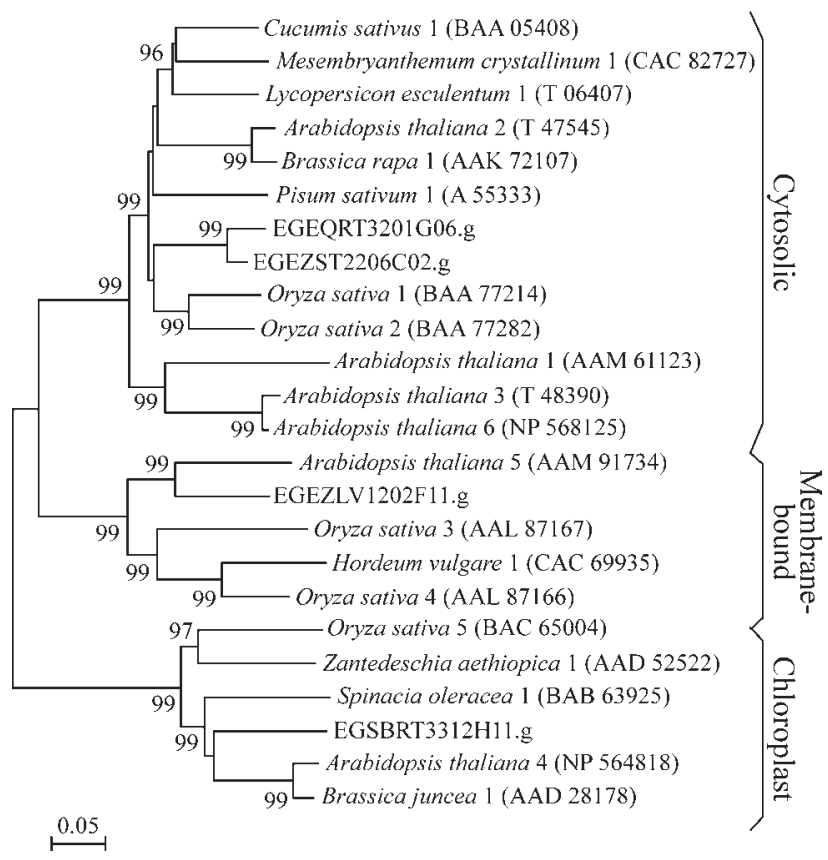

Figure 5 - Phylogenetic tree of MDHAR proteins. Phylogenetic analyses were conducted as described in Figure 2.

EGSBRT3312H11.g, which encodes a putative chloroplastic isoform, is 5'-incomplete and the N-terminus of its protein is absent. The analysis of the phylogenetic tree (Figure 5) clearly shows the clustering of five proteins containing one or more putative transmembrane domains. This group is more closely related to the cytosolic isoforms when compared to the chloroplastic isoforms. In fact, the membrane bound branch grouped with the cytosolic branch. Unfortunately, no component of the membrane-bound group was previously characterized in relation to its subcellular localization. However, the phylogenetic tree shows high interior branch test values (Figure 5).

DHAR catalyzes the reduction of dehydroascorbate to ascorbate, using glutathione as reducing substrate (Figure 1). Cytosolic DHAR encoding cDNAs from wheat, rice, tomato, and arabidopsis, and chloroplastic cDNAs from spinach were previously described (Chen et al., 2003; Shimaoka et al., 2000). We have identified three Eucalyptus clusters encoding DHAR isoforms, but two of them do not represent full-length transcripts. Subcellular prediction and phylogenetic analyses show that two clusters encode putative cytosolic isoforms and the third one encodes a putative chloroplastic isoform (Table 1 and Figure 6). The $A$. thaliana glutathione S-transferase protein (accession number NP198937) was included in the phylogenetic analysis as an out-group. The two Eucalyptus cytosolic DHAR encoding clusters (EGEPFB1249A11.g and EGQHST6232 H12.g) grouped with previously described DHAR cytosolic isoforms (Figure 6). Similarly, the chloroplastic encoding cluster (EGEZSL7225G03.g) also grouped with previously described chloroplastic isoforms. Moreover, the putative chloroplastic protein de- 


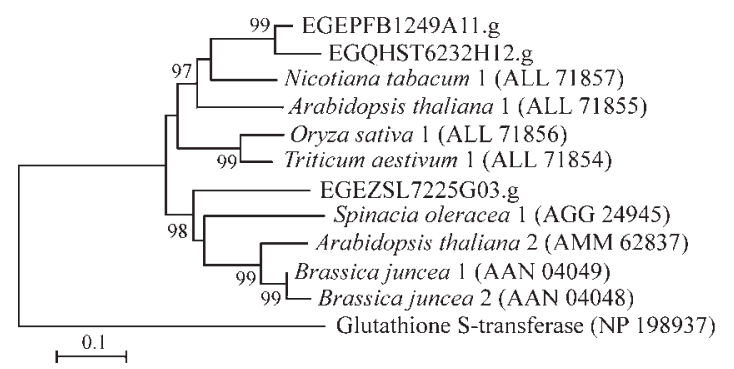

Figure 6 - Phylogenetic tree of DHAR proteins. Phylogenetic analyses were conducted as described in Figure 2.

duced from this cluster harbors an N-terminal extension region characteristic of chloroplastic sorting peptide sequences, which is $65 \%$ similar to the spinach chloroplastic isoform sequence previously described (Shimaoka et al., 2000).

In the final step of the ascorbate-glutathione cycle, GR catalyzes the regeneration of glutathione disulphide to glutathione, using NADPH as the electron donor (Figure 1). The cDNAs corresponding to cytosolic GRs from pea (Stevens et al., 1997) and Brassica rapa (Lee et al., 1998), and chloroplastic GR cDNAs from pea (Creissen et al., 1995) and Arabidopsis (Kubo et al., 1993) were previously reported. May and Soll (2000) proposed that the $\mathrm{N}$-terminal sorting peptide present in the chloroplastic GR isoforms may interact with 14-3-3 proteins, leading them to chloroplast translocation. Two clusters encoding GR were identified in FORESTs database (Table 1). Both phylogenetic (Figure 7) and subcellular prediction analyses indicate that the complete cluster EGEQRT3201 H09.g encodes a putative cytosolic isoform. On the other hand, the 3'-incomplete cluster EGEQRT6002D01.g corresponds to a potential chloroplastic isoform and is preceded by a putative N-terminal chloroplastic sorting peptide similar to that found in pea chloroplastic GR (accession number P27456). The A. thaliana thioredoxin reductase protein (accession number NP195271) was included in the phylogenetic analysis as an out-group (Figure 7).

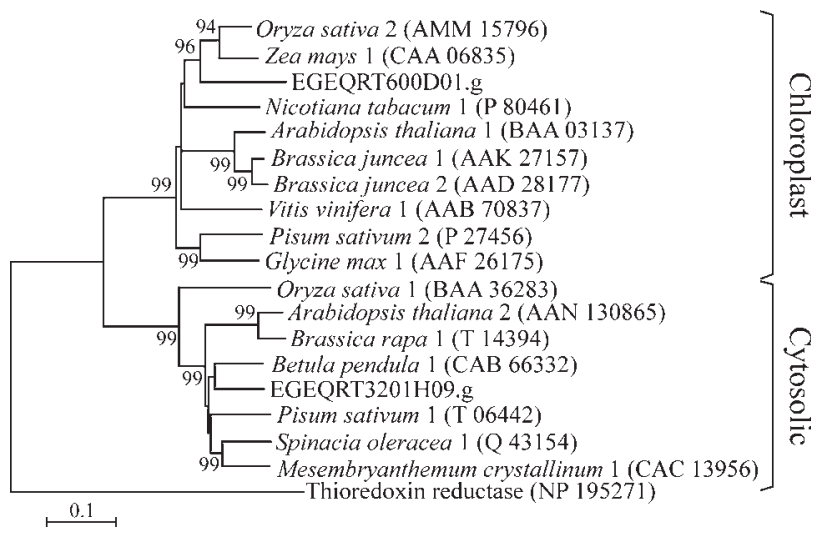

Figure 7 - Phylogenetic tree of GR proteins. Phylogenetic analyses were conducted as described in Figure 2.
The ascorbate-glutathione cycle is particularly important for the maintenance of chloroplast redox homeostasis. Likewise, this cycle also operates in the cytoplasm (Alscher et al., 1997; Noctor and Foyer, 1998). We identified Eucalyptus clusters encoding cytosolic and chloroplast isoforms for all enzymes that catalyze key steps of the cycle (Table 1). Additionally, the structure of all phylogenetic trees reveals a clear divergence between chloroplastic and non-chloroplastic isoforms (Figure 4 to 7). Two main groups are distinguished and show high Interior Branch Test values. One group includes all proteins localized or predicted to be in the chloroplast. Proteins that are not sorted to the chloroplast are grouped in a second branch, formed by proteins localized or predicted as soluble in the cell cytosol or anchored to membranes. These results confirm the presence of a complete ascorbate-glutathione cycle in both Eucalyptus cytosol and chloroplasts.

As a preliminary investigation of the expression pattern of the genes described here, the different ESTs libraries that were constructed for the FORESTs project were analyzed for the presence of those clusters. The distribution and frequency of each cluster is presented in Table 2. No clear distribution pattern of the clusters encoding ascorbate-glutathione cycle enzymes was observed. A cluster encoding a putative cytosolic DHAR isoform (EGQHST6232H12.g) is the only one present in a single library (Table 2). In general, the cytosolic isoforms of the different enzymes that participate in this cycle were more frequently represented in the libraries than the chloroplastic isoforms. Furthermore, a higher number of clusters encoding putative cytosolic isoforms was observed in comparison to the chloroplastic ones. Taken together, the results may indicate an important role of the cytosolic cycle in the control of ROS levels in Eucalyptus.

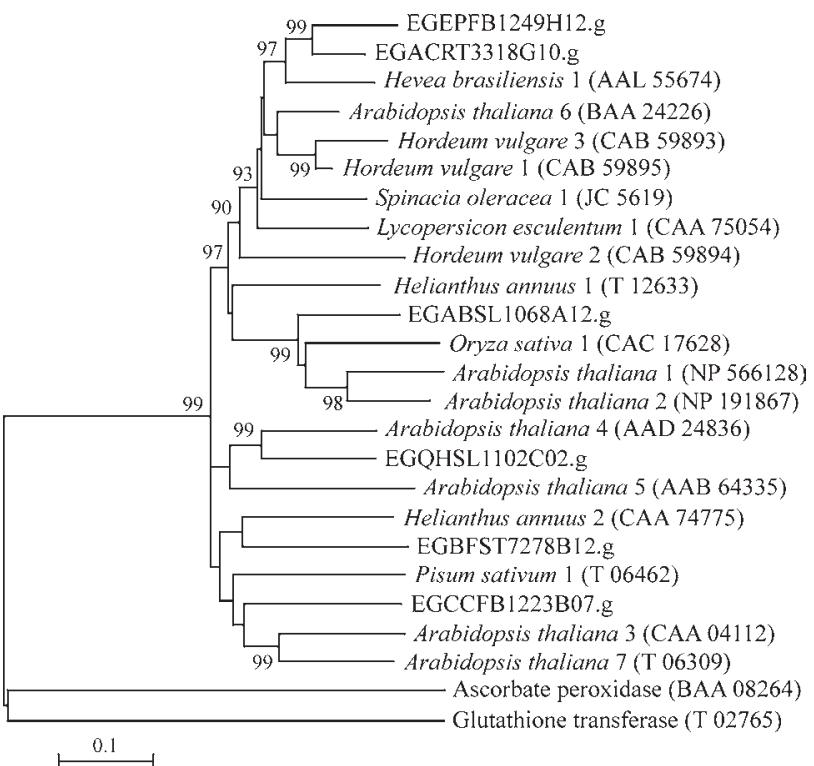

Figure 8 - Phylogenetic tree of PhGPx proteins. Phylogenetic analyses were conducted as described in Figure 2. 


\section{Phospholipid glutathione peroxidase clusters}

Phospholipid hydroperoxide glutathione peroxidases catalyze the reduction of lipid hydroperoxides to alcohol using glutathione as the electron donor. A cytosolic PhGPx encoding cDNA from tomato (Depège et al., 1998) and chloroplastic PhGPx encoding cDNAs from pea and Arabidopsis (Mullineaux et al., 1998) were previously reported. Furthermore, citrus PhGPx has been purified and the enzyme activity has been determined (Beeor-Tzahar et al., 1995). We have identified six Eucalyptus clusters encoding PhGPx. The subcellular prediction analysis indicates that three of them encode putative cytosolic isoforms and three correspond to putative chloroplastic isoforms (Table 1). Therefore, the Eucalyptus PhGPx is the largest phospholipid hydroperoxide glutathione peroxidase gene family reported for plants. The N-terminal section of the putative chloroplastic isoforms encodes a recognizable chloroplast transit peptide, similar to those found in pea and Arabidopsis chloroplastic PhGPx (Mullineaux et al., 1998). The PhGPx phylogenetic tree confirms the prediction that these clusters encode putative PhGPx, since the Eucalyptus isoforms grouped with other previously described PhGPx proteins (Figure 8). Rice ascorbate peroxidase (accession number BAA08264) and glutathione S-transferase (accession number T02765) were included in the phylogenetic analysis as out-groups.

The PhGPx clusters distribution among the FORESTs libraries are described in Table 2. The most frequent PhPGx cluster among the libraries corresponds to a putative chloroplastic isoform (EGEPFB1249H12.g). In fact, the putative chloroplastic PhGPx-encoding clusters are more represented than the cytosolic ones.

\section{Conclusions}

The data discussed here may contribute to the understanding of the role of different genes of the antioxidant metabolism in plants. Our results reveal the presence of a complete ascorbate-glutathione cycle in the two Eucalyptus cell compartments, cytosol and chloroplasts.Also, some particularities specific to this plant were observed: the large gene family encoding PhGPx and the broad expression of SOD isoforms, suggesting the crucial role of these enzymes in ROS scavenging. Our results also indicate a major importance of catalases in Eucalyptus antioxidant metabolism.

The in silico preliminary expression analyses indicate that a comprehensive study of antioxidant gene expression should provide new insights into the role of the biological diversity of antioxidant enzymes in Eucalyptus, as revealed in this study.

\section{References}

Alscher RG, Donahue JL and Cramer CL (1997) Reactive oxygen species and antioxidnats: Relationships in green cells. Physiol Plant 100:224-233.
Altschul SF, Gish W, Miller W, Myers EW and Lipman DJ (1990) Basic local alignment search tool. J Mol Biol 215:403-410.

Beeor-Tzahar T, Ben-Hayyin G, Holland D, Faltin Z and Eshdat YA (1995) A stress-associated citrus protein is distinct plant phospholipid hydroperoxide glutathione peroxidase. FEBS Lett 366:151-1555.

Bowler C and Fluhr R (2000) The role of calcium and activated oxygen species as signals for controlling cross-tolerance. Trends Plant Sci 5:241-246.

Chen Z, Young TE, Ling J, Chang S and Gallie DR (2003) Increasing vitamin $\mathrm{C}$ content of plants through enhanced ascorbate recycling. Proc Natl Acad Sci USA 100:3525-3530.

Creissen G, Reynolds H, Xue Y and Mullineaux P (1995) Simultaneous targeting of pea glutathione reductase and of a bacterial fusion protein to chloroplasts and mitochondria in transgenic tobacco. Plant J 8:167-175.

Dat J, Vandenabeele S, Vranova E, Van Montagu M, Inzé D and Van Breusegem F (2000) Dual action of the active oxygen species during plant stress responses. Cell Mol Life Sci 57:779-795.

Depège N, Drevet J and Boyer N (1998) Molecular cloning and characterization of tomato cDNAs encoding glutathione peroxidase-like proteins. Eur J Biochem 253:445-451.

Eshdat Y, Holland D, Faltin Z and Ben-Hayyim G (1997) Plant glutathione peroxidases. Physiol Plant 100:234-240.

Fridovich I (1998) Oxygen toxicity: A radical explanation. J Exp Bot 201:1203-1209.

Frugoli JA, Zhong HN, Nuccio ML, McCourt P, McPeek MA, Thomas TL and McClung CR (1996) Catalase is encoded by a small multigene family in Arabidopsis thaliana (L.) Heynh. Plant Physiol 112:327-336.

Grantz AA, Brummell DA and Bennett AB (1995) Ascorbate free radical reductase mRNA levels are induced by wounding. Plant Physiol 108:411-418.

Havir AE and McHale NA (1987) Biochemical and developmental characterization of multiple forms of catalase in tobacco leaves. Plant Physiol 84:450-455.

Higgins DG and Sharp PM (1988) CLUSTAL: A package for performing multiple sequence alignment on a microcomputer. Gene 73:237-244.

Hirokawa T, Boon-Chieng S and Mitaku S (1998) SOSUI: Classification and secondary structure prediction system for membrane proteins. Bioinformatics 14:378-379.

Hossain MA, Nakano Y and Asada K (1998) Monodehydroascorbate reductase in spinach chloroplasts and its participation in generation of ascorbate for scavenging hydrogen peroxide. Plant Cell Physiol 25:385-395.

Iwamoto M, Maekawa M, Sato A, Higo H and Higo K (1998) Evolutionary relationship of plant catalase genes inferred from exon-intron structures: Isozymes divergence after the separation of monocots and dicots. Theor App Genet 97:919.

Jespersen HM, Kjaersgard IV, Ostergaard L and Welinder KG (1997) From sequence analysis of three novel ascorbate peroxidases from Arabidopsis thaliana to structure, function and evolution of seven types of ascorbate peroxidase. Biochem J 326:305-310.

Jimenez A, Hernandez JA, Del Rio LA and Sevilla F (1997) Evidence for the presence of the ascorbate-glutathione cycle in mitochondria and peroxisomes of pea leaves. Plant Physiol 114:275-284. 
Jones AM (2001) Programmed cell death in development and defense. Plant Physiol 125:94-97.

Karpinska B, Karlsson M, Schinkel H, Streller S, Su K, Melzer M and Wingsle G (2001) A novel superoxide dismutase with a high isoelectric point in higher plants. Expression, regulation, and protein localization. Plant Physiol 126:1668-1677.

Kliebenstein DJ, Monde RA and Last RL (1998) Superoxide dismutase in Arabidopsis: An ecletic enzyme family with disparate regulation and protein localization. Plant Physiol 118:637-650.

Kubo A, Sano T, Saji H, Tanaka K, Kondo N and Tanaka K (1993) Primary structure and properties of glutathione reductase from Arabidopsis thaliana. Plant Cell Physiol 34:1259-1266.

Kumar S, Tamura K, Jakobsen IB and Nei M (2001) MEGA2: Molecular Evolutionary Genetics Analysis software. Bioinformatics 17:1244-5.

Lee H, Jo J and Son D (1998) Molecular cloning and characterization of the gene encoding glutathione reductase in Brassica campestris. Biochim et Biophys Acta 1395:309-314.

Madhusudhan R, Ishikawa T, Sawa Y, Shigeoka S and Shibata H (2003) Characterization of an ascorbate peroxidase in plastids of tobacco BY-2 cells. Physiol Planta 117:550-557.

May T and Soll J (2000) 14-3-3 proteins form a guidance complex with chloroplast precursor proteins in plants. Plant Cell 12:53-63.

McClung CR (1997) Regulation of catalases in arabidopsis. Free Rad Biol Med 23:489-496.

Mullen RT and Trelease RN (2000) The sorting signal for peroxissomal membrane-bound ascorbate peroxidase are within its C-terminal tail. J Biol Chem 275:16337-16344.

Mullineaux PM, Karpinski S, Jimenez A, Claery SP, Robinson C and Creissen GP (1998) Identification of cDNAs encoding plastid-targeted glutathione peroxidase. Plant J 13:375-379.

Murthy SS and Zilinskas BA (1994) Molecular cloning and characterization of a DNA encoding pea monodehydroascorbate reductase. J Biol Chem 269:31129-31133.

Nakai K and Horton P (1999) PSORT: A program for detecting the sorting signals of proteins and predicting their subcellular localization. Trends Biochem Sci 24:34-35.

Netto LE (2001) Oxidative stress response in sugarcane. Gen Mol Biol 24:93-102.

Ni W, Turley R and Trelease R (1990) Characterization of a cDNA encoding cottonseed catalase. Biochim Biohys Acta 1049:219-222.

Nicholas KB, Nicholas HB and Deerfield DW (1997) GeneDoc: Analysis and visualization of genetic variation. EMBNEW.NEWS 4:14.
Noctor G and Foyer CH (1998) Ascorbate and glutathione: Keeping active oxygen under control. Annu Rev Plant Physiol Plant Mol Biol 49:249-79.

Obara K, Sumi K and Fukuda H (2002) The use of multiple transcription starts causes dual targeting of Arabidopsis putative monodehydroascorbate reductase to both mitochondria and chloroplasts. Plant Cell Physiol 43:697-705.

Patterson WR and Poulos TL (1995) Crystal structure of recombinant pea cytosolic ascorbate peroxidase. Biochemistry 34:4331-4341.

Sano S and Asada K (1994) cDNA cloning of monodehydroascorbate radical reductase from cucumber: A high degree of homology in terms of amino acid sequence between this enzyme and bacterial flavoenzymes. Plant Cell Physiol 35:425-437.

Scandalios JG (1965) Subunit dissociation and recombination of catalase isozymes. Proc Natl Acad Sci 53:1035-1040.

Scandalios JG (1997) Molecular genetics of superoxide dismutase in plants. In: Scandalios JG (ed) Oxidative Stress and Molecular Biology of Antioxidant Defenses. Cold Spring Harbor Laboratory Press, Cold Spring Harbor, pp 527-568.

Scandalios JG (2002) The rise of ROS. Trends Biochem Sci 27:483-486.

Scandalios JG, Guan L and Polidoros AN (1997) Catalases in plants: Gene structure, properties, regulation, and expression. In: Scandalios JG (ed) Oxidative Stress and Molecular Biology of Antioxidant Defenses. Cold Spring Harbor Laboratory Press, Cold Spring Harbor, pp 343-406.

Schinkel H, Hertzberg M and Wingsle G (2001) A small family of novel $\mathrm{CuZn}$-superoxide dismutase with high isoelectric points in hybrid aspen. Planta 213:272-279.

Shigeoka S, Ishikawa T, Tamoi M, Miyagawa Y, Takeda T, Yabuta Y and Yoshimura K (2002) Regulation and function of ascorbate peroxidase isozymes. J Exp Bot 53:1305-1319.

Shimaoka T, Yokota A and Miyake C (2000) Purification and characterization of chloroplast dehydroascorbate reductase from spinach leaves. Plant Cell Physiol 41:1110-8.

Stevens RG, Creissen GP and Mullineaux PM (1997) Cloning and characterisation of a cytosolic glutathione reductase cDNA from pea (Pisum sativum L.) and its expression in response to stress. Plant Mol Biol 35:641-654.

Van Camp W, Inzé D and Van Montagu M (1997) The regulation and function of tobacco superoxide dismutase. Free Radic Biol Med 23:515-520.

Willekens H, Langebartels C, Tiré C, Van Montagu M, Inzé D and Van Camp W (1994) Differential expression of catalase genes in Nicotiana plumbaginifolia (L.). Proc Natl Acad Sci USA 91:10450-10454.

Associate Editor: Carlos F.M. Menck 